\title{
Management of Post-Purchase Regret
}

\author{
Melika Ben M’Barek and Abderrazak Gharbi
}

University of Tunis El Manar, Tunisia

\begin{abstract}
The purpose of this article consists in exploring the regulation strategies of post purchase regret in the consumer. A qualitative approach was used via in-depth interviews, and focus groups were conducted.

The results obtained allowed us to show that the consumer is often motivated to regulate his regret to restore a psychological balance that was broken. These regulating strategies of regret may be focused either on the objective, the decision, the alternative or on the feeling.
\end{abstract}

Key words: regret - regulating- objective- feeling- alternative- decision.

\section{Introduction}

Psychologists and studies on consumer behavior provided extensive evidence that emotions influence behaviors (Baggozi and al, 2000). Researchers no longer adopt a dimensional approach; they only consider the overall satisfaction to understand the consumer's behavior. Currently, they are more interested in the specificities of emotional experiences to better understand consumer's behavior that becomes very changeable.

Regret is, among other things, a specific emotion that has a profound impact on decision making. It guides behaviors since people often tend to avoid it.

Recently, research has shown that regret is not only an affective reaction to the poor results of decision, but also a powerful force that motivates and directs our behaviors. Regret is not pleasant, it negatively affects our well being, it guides our behaviors and we are motivated to avoid it, and to manage it if it appears.

Several research works were interested in regret, but very few of them have explored the regulating strategies of this painful emotion.
In this paper, the researchers will present the contribution of previous research on regulating strategies of regret developed. Then they will explore through qualitative research the different ways consumers manage and regulate their post-decisional regrets.

\section{Literature Review}

Regret is a cognitive emotion which people are motivated to regulate the way they regulate any other emotion. They engage in self regulation process. Self-regulatory processes are evident in all aspects of people's behavior, and are exerted in any effort by people to alter their responses. Self-regulation refers to the process by which people initiate, adjust, interrupt, terminate, or otherwise alter actions to promote attainment of personal goals, plans, or standards (e.g., Carver and Scheier, 1998). The process of selfregulation involves several important components: having clear standards of how things should be, comparing one's actual state to a desired state indicated by the standards and overriding responses and bringing about a desired change in case the current state falls short of the standards (Carver and Scheier, 1998).

Copyright (C) 2012 Melika Ben M'Barek and Abderrazak Gharbi. This is an open access article distributed under the Creative Commons Attribution License unported 3.0, which permits unrestricted use, distribution, and reproduction in any medium, provided that original work is properly cited. Contact author: Melika Ben M’Barek E-mail: melikabm@gmail.com 
In principle, the negative consequences of regret can be regulated in two ways:

Individuals can either actively change the conditions that led to regret;

Or internally adapt the behavior of regret by adjusting their perceptions of responsibility and control of regret.

The two ways of doing things facing regret are coherent with the distinction between "primary control and secondary control" originally introduced by Rothban and al (1982) and reformulated in a developed context by Hechausent and Schulz (1995, 1996).

The primary control strategies are directed to produce a change in the external world. And secondary control strategies are directed towards the optimization of motivational resources and perceptions (related to oneself).

Thus, individuals engage in reparation mechanisms to manage their regret and restore their psychological well being and that is through feelings, actions, trends of actions etc...

Reparation strategies differ according to the felt emotion. Responses related to regret are more oriented towards oneself, while those related to dissatisfaction are more oriented towards others. The responses related to regret tend to be internal and silent, in the presence of introspective emotions such as shame, while those related to dissatisfaction tend to be oriented outward in the presence of emotions such as anger and frustration (Aron, 2000).

Zeelenberg and Pieters (2007) stated that the regulation of regret is indeed secondary to the regulation of behavior. In other words, they clearly specified that people regulate their regret in order to regulate their behaviors.

Zeelenberg and Pieters (2007) cited in their regret regulation theory a list of strategies for effective regret and anticipated regret.
They note that the main regulating strategies are the same for both regret felt as well as anticipated regret, in spite of the fact that the specific mechanisms differ.

These strategies are classified into four groups: strategies focused on the objective; strategies focused on the decision; strategies focused on the alternative and strategies that focus on the feeling.

\section{Strategies Focused on the Objective: Reducing the Level of the Objective}

The regulating strategy focused on the objective is a strategy regulating emotions in general and regret in particular. Which does not remain so obvious to be applied, but they nevertheless introduced it in the second version of their theory.

The regulating strategy focused on the objective is related to the notion of "Bracing for loss" according to which the consumer expects the worst as to avoid the potential negative emotions (Shepperd and al, 2000; Van Dijk and al, 2003). Zeelenberg and Pieters (2007) suggest that the decline in the level of the objective is a regulating strategy that deserves to be discussed further in future research.

\section{Strategies Focused on Decision}

Strategies for regulating regret focused on decision aims at both the decision process and the results obtained. There are three strategies: cancelling the decision, justifying the decision and denying one's responsibility for the decision.

First, it is demonstrated that when the product is not optimal or does not meet the desired expectations, consumers are likely to cancel the decision and that by returning the product or shifting to another brand, another supplier or another product is chosen the next time. (Zeelenberg and Pieters, 1999; 2004; Zeelenberg and al, 1998).

Therefore, regret can be beneficial and can help the consumer meet his/her needs and improve his/her wellbeing. 
Then to protect himself from regret both before and after decision making, the individual tends to justify his choice. He tries to provide good reasons to prove that his choice was good given the circumstances.

Finally, and starting with the close relationship between regret and responsibility, denying the perceived responsibility of the error is an obvious solution to mitigate the regret felt. (Zeelenberg and Pieters, 2007)

\section{Strategies Focused on the Alternative}

The regulating strategies of regret focused on alternative are of the order of two factors; namely, either changing the chosen alternative or its revaluation by generating counterfact type "even if ..." for example, "even though I chose differently the result could have been the same". Thus, engaging in downward counterfactual thoughts could make regret less intense or even make it disappear.

If engaging in this kind of counterfactual thoughts is not possible, then they neglect the rejected alternatives or simply abstain from using them as reference in the comparisons.

\section{Strategies Focused on Feeling}

Zeelenberg and Pieters (2007) postulate that acting on the feeling through a psychological work of reparation or by canceling regret at all is also a way of managing regret.

The psychological work of reparation consists in taking decisive action to be convinced, cancelling or compensating a negative result (and Medevec Gilovich, 1995).

Gilovich and Medevec (1995) described two forms of psychological reparation which the decision maker can take to mitigate regret.

First, identifying the good sides, the individual can convince himself, for example, that he learned well from regretted mistakes, or by thinking that things might have been worse.

Second, people may engage in strategies of dissonance reduction. Thus, decision makers regulate their regret by focusing on the positive rather than negative sides and think about the future instead of the past.

Moreover, some decision makers express some reluctance in recognizing its existence. So, they actively seek to deny it or remove it. These people think that one should never regret anything.

\section{Research Methodology}

The purpose of this work of research is purely exploratory. It aims at understanding and determining the regulating strategies of regret. Besides, it was given little attention by previous works. It is for these reasons that the researchers have opted for a purely qualitative approach through in-depth interviews and focus groups.

Our sample is structured as follows:

- Four focus groups: two groups of men and two groups of women, two groups of young people and two groups of aged people while providing different socioprofessional categories. Each group contains eight persons. Each discussion takes around 90minutes.

- Fifteen individual interviews: this number was determined according to the principle of saturation. The duration of each interview was 45 minutes.

Several methods of direct as well as projective questioning via the technique of scenarios, free associations, the analogical method and the technique of sentence and story completing were used. The projective techniques are very important and helped to get a lot of important information.

The researchers performed full transcription of the respondents' discussions; and to facilitate the operations of categorization, coding, quantification and construction of analysis grids, 
qualitative data processing software, NVIVO 8, was used.

The method of analysis used is thematic analysis. Inter-interviews and inter-groups analyses were performed in order to compare the respondents' viewpoints and identify the general trends in terms of behaviors and reactions vis-à-vis the topics discussed. The results obtained will be presented in the following section.

\section{Research Results}

Remember that the primary objective of this research consists in exploring the regulating strategies of regret which was given but little attention in previous research. These strategies were classified in this article as in the works of Zeelenberg and Pieters (2007).

\section{Strategies Focused on the Objective}

\section{Reducing the Objective Level}

The meaning units identified in this category were not redundant but their content was still very important. Respondents often implicitly suggested that they reduce the level of the objective to be achieved by a decision to regulate their post-purchase regret. Indeed, when the objective is reduced, there is a strong chance the evaluation of the decision will be positive, hence lowering the feeling of regret. Response type is: "... in fact he should better say to himself my objective was still to have a perfect design, an affordable price, I receive my emails on time ... so if the image quality is not perfect there is no problem after all I use my digital camera to take pictures ... ".

Respondents also often set the example "of brace to the loss," that is to say, the more they expect the loss, the less they regret. "... When we decide we must be mentally prepared to gain and loss ...". But this kind of thought often takes place before decision-making and not during the postpurchase evaluation phase.

\section{Strategies Focused on Decision}

To manage their regrets, individuals can act on the decision. This is achieved either by acting on the result or on the process by which the decision is made. The corpus analysis allowed to infer that respondents regulate their post-purchase regret by canceling the decision either by justifying or denying the responsibility altogether.

\section{Cancelling the Decision}

Respondents implicitly or explicitly pointed out that to manage their post-purchase regret, cancelling the decision is a very effective way to do so. Indeed, they say that when the decision is canceled for example by returning the product or exchanging it with another product that is not substitutable, they supress regret since the error has been rectified. In this case, the psychological equilibrium is restored, regret felt was a lesson to improve future decisions and this painful sensation was regulated: "... I regretted the bag, so I returned it to the store, I exchanged it with a dress and although I paid an extra I was happy anyway..."

Respondents often said that cancelling the decision is very much regulating post purchase regret, but it is rarely possible and sometimes costs a big indemnity.

\section{Lustification of the Decision}

This category was frequently mentioned by respondents. It is the most pronounced regulating strategy by the interviewees (51\% pronounced it). Indeed, they say that if they succeed to justify their erroneous perceived decisions, the intensity of regret felt is reduced. 
Particularly when they justify their decisions, they reduce the perceived responsibility of the error. Thus, the feeling of self-blame will be reduced and the regret will be regulated. Interviewees may also justify the process by which the decision is made and they often try to justify themselves with arguments type: "they had no time to take the right decision," "they were obliged to," "they did not have enough money "," they chose a known brand "etc....

Therefore if consumers manage to justify the decision or the process by which the result was reached, the individual will succeed in reducing the feeling of selfblame and the psychological discomfort felt. This type of strategy does not totally eliminate regret felt because that is performed in an internal way. The alternative is not affected and the error is not corrected.

\section{Denying one's Responsibility for the Decision}

This category is also redundant in the overall corpus (35\%). Denying one's responsibility is a way to reduce regret. Respondents often try to deny responsibility for the decision, and in turn throws the responsibility on others. "... It's not my fault ..."

Indeed laying the responsibility on someone else will mitigate the sensation of self-blame and regret felt. Respondents often throw the responsibility on the vendor or the person accompanying them when purchasing "... It is the saleswoman who misled me, she insisted and she convinced me ..." "... it was my mother's fault, I told her if she did not hurry me I would have seen well and I would not have been had ... ".

Denying responsibility relieves and reduces the feeling of regret felt. This relationship between regret and responsibility was so obvious and important so much that the interviewees explicitly pointed out that sometimes they prefer to entrust decision making to someone else or to be surrounded with many experts in order to reduce the responsibility of the error and regret felt afterwards.

\section{Strategies Focused on the Alternative}

\section{Reversing the Decision: Shift to the Rejected Alternative}

Previous researches proved that the reversibility of the decision helps mitigate post-purchase regret. Indeed, respondents try to reverse the decision as soon as they receive information in favor of the rejected option. They try to cancel the decision by returning the product for example to shift to the rejected alternative. "... I woke up early and I went to the store to return the pants I bought. I exchanged it with the other one I believe to be better and I was very happy when the saleswoman did not refuse ..."

This strategy definitely eliminates the regret felt since the error is rectified and the state of psychological equilibrium is restored. However, this kind of action is not obvious because it depends on several external factors, namely whether the decision is reversible or not, whether the supplier agrees to reverse the decision or not, whether the information about the rejected alternative is provided immediately after taking the decision or not etc...

\section{Reassessing the Quality of the Alternative}

Some interviewees mentioned in a spontaneous or assisted way that they reassess the quality of the chosen alternative if no possibility of exchange or cancellation is possible. The analysis of the transcripts show that they try to be convinced by their choices by only considering positive sides and overestimating the chosen option through generating descending counterfact.

Thus, respondents engage in counterfactual reasoning in order to evaluate the chosen alternative in a more positive way. They sometimes try to undervalue the rejected alternatives or to suppress them altogether. Response type: "... she tries to convince herself that everything is ok" "... she 
will say: oh in the morning I do not eat toast because I do not eat bread why did I buy it? Ah in the evening I feel like eating something sweet I'll take a spoon of it". Also sometimes they try to ignore the alternatives rejected and just focus on the strengths of their choice «... I avoid comparisons simply, when I bought I stop looking back" ... ».

Furthermore, sometimes they try to ignore the rejected alternatives and just focus on the strengths of their choices." I simply avoid comparisons, this is it I bought I'm not seeking anymore...."

\section{Abandoning the Alternative}

Respondents frequently mentioned that abandoning the chosen alternative helps to mitigate post-purchase regret. Indeed, they have often pointed out the fact that getting rid of the chosen alternative helps reduce the intensity of post-purchase regret. This is a way that allows forgetting the error.

Sometimes to restore the state of psychological balance, they try to buy another alternative even non-substitutable. "Yes I went to buy another one in its place ..." "... the product is put out of our field of vision; we buy something else as a distraction ".

\section{Strategies Focused on the Feeling}

The analysis of the meaning units allows inferring that respondents can regulate their post-purchase regret by acting on the feeling. They try to change moods, to engage in a psychological work of reparation, to suppress or cancel regret and via religion.

\section{Psychological Work of Reparation}

The analysis of meaning units brought out in this category allows inferring that the interviewees try to perform an internal psychological work of reparation and try to have themselves convinced that no error was made. They try to have themselves convinced that they made a good choice by undervaluing the rejected alternative and by overvaluing the chosen alternative. They try to consider the positive sides of the decision only even if it is negative. "... I try to get myself convinced that I took the right decision..."

People try then to look the positive sides of their decisions and to neglect the negative aspects. "...It is true that the living room did not perfectly suit with the type of decoration of my house but it is an excellent Italian style, it opens up to become larger and round, it is still very good quality...". They try to have themselves convinced that they made the good choice "it is true that glasses were too expensive but they are excellent, Bvlgary brand is the best..."

Or simply respondents try to have themselves convinced that in spite of the error it is not the last time they take a decision and they are learning for sure: “...after all I learned a good lesson...", it is the education aspect of regret.

What we could conclude is that psychological works of reparation depend much on the individual's personality, the more the individual has a strong personality and self-confidence, the more he/she can manage his/her regret by engaging into often positive psychological works of reparation.

\section{Suppressing or Cancelling Regret}

This category, though it was not frequently present in the overall corpus, still proved very important and is worth being pointed out. Some of the interviewees mentioned they did not regret anything in their lives and that they have a strong capacity to escape any failure. "...as for me I regret nothing...", "...I don't recall I ever regretted anything...".

Nevertheless, implicitly via projective techniques they spoke about regret. Therefore, for these persons regret can be a symbol of weakness which they seek to hide or sometimes a much embarrassing feeling which they try to internally cancel.

Suppressing or cancelling regret is in this case a way to regulate this painful sensation. Yet, this regulation strategy 
often remains difficult and depends on the individual's personality.

\section{Changing Mood}

It was often mentioned that changing mood is also a way to regulate regret. Indeed, regret is perceived as being a state of psychological uneasiness accompanied with bad mood. "...he tries to take holidays to forget this bad experience; he goes out with friends, he has a break...", “...to enjoy oneself, doing sport, going out with friends, to simply forget...". Therefore the fact of changing mood, of enjoying oneself, etc. is a way to forget the failure in general and regret in particular.

\section{Religion}

This category was frequently mentioned especially by adult respondent males and females. These respondents often mentioned that being a good believer in God is a very good way. Thus, religion and faith is a way to regulate one's regret.

Respondents have themselves convinced that the decision already made is the one God chose for them and they must be convinced that it is their fate. It is, among other things, a way to deny the responsibility of the choice. Responses type: "...those who are much believing, who know that it is God Who offered it to them do not regret...", and not to regret big decisions people pray, and it is a very reassuring way especially with regard to big decisions "...when we pray, we strangely feel satisfied it does not matter if it is granted or not...".

\section{Discussion and Conclusion}

As a result to this qualitative analysis, it has been shown that people are often motivated to regulate their post-decisional regrets. It is a painful feeling which hides behind an imbalance and psychological discomfort sometimes unbearable.

- People can engage in activities focused on the objective to manage the level of regret felt by lowering the level of objectives fixed beforehand. As it was mentioned in the theory of Zeelenberg and Pieters (2007), this exploratory study confirms that lowering the level of objectives is a way to reduce the level of the objective to be reached by a decision to regulate their post-purchase regret.

- Regulation of regret can also be made through strategies focused on the decision: To focus on the decision by canceling it altogether or justifying it by denying the responsibility of the error allows managing the level of regret felt. These strategies of regulation were mentioned only in the theory of regret regulation by Zeelenberg and Pieters (2007). The current results converge to the assumptions of Zeelenberg and Pieters (2007).

- People can also focus on the alternative even to mitigate their post decisional regret. These results converge and enrich the propositions of Zeelenberg and Pieters (2007). Indeed, it was concluded that the regulation strategies of regret focused on the alternative consist in reversing or changing the chosen alternative, re-evaluating its quality but also abandoning it altogether. The latter was frequently present in the respondents' speeches as an effective means to reduce the regret intensity felt.

In most cases, the immediate reaction to regulate regret is often to shift to the rejected alternative. For this reason, people often demand reversibility through guarantees and even accept to pay an indemnity as this will allow them to protect themselves and face regret.

Moreover, and in order to mitigate their regret people try to re-evaluate the chosen alternative to minimize the gap between the chosen alternative and the rejected one. They engage in counterfactual thinking in order to highlight the benefits of the chosen alternative and disadvantages of the rejected alternative. These results were only mentioned, to our knowledge, by Zeelenberg and Pieters as (2007).

However, and as a result to this thematic analysis, it seems that abandoning the 
alternative and sometimes buying another one that is not necessarily the rejected alternative is a way to manage one's regret So, it is a way to forget failure. This research is the first, to our knowledge, to cite this regulation strategy of regret.

- Strategies for regulation of regret can also be internal and focused on feeling. The researchers have shown that when no action of return, of cancellation or change of decision is possible, a psychological work of reparation can serve to restore this psychological balance. So people can have themselves convinced that no error was committed. They adopt a positive attitude, considering only the positive aspects of the decision. Similarly, they can have themselves convinced that even if it was a negative experience, it is thanks to negative experiences that we learn to improve regret. This result was mentioned in the theory of regret regulation by Zeelenberg and Pieters (2007).

The extensive analysis of the respondents' speeches has also demonstrated that suppressing or cancelling regret is a way to regulate regret. Certain individuals assume that one should not regret anything in life and that experiences are all relevant to build up the future (Zeelenberg and Pieters, 2007).

Moreover, two new strategies can complement the two previous regulation strategies of regret focused on feeling proposed by Zeelenberg and Pieters (2007).

First, it was mentioned that people sometimes seek, after an experience of regret, a change of mood to forget their negative experiences. This will help regulate regret in them, for it is a way to overcome the past and minimize counterfactual thoughts. This regulation strategy of regret was only mentioned in this work of research.

Then, regret can be reduced as soon as we are convinced that the result is part of destiny and that it is probably beneficial somewhere in our lives. Those who believe in this idea can easily overcome their regrets. This means to regulating regret is mainly based on the belief in God and is part of some principles of the Islamic religion. It was not mentioned in any previous research but it was very apparent in respondents' speech.

Thus, it is stated in this article that regret is a painful feeling which causes great psychological imbalance and people are often motivated to avoid it and when they fail, they engage in a reparative behavior via different regulating strategies to reduce the intensity of regret felt. The researchers tried to confirm and complete the list of regulation strategies of regret presented by Zeelenberg and Pieters (2007) and which was deemed incomplete by its founders. However, the researchers noticed that these strategies differ from one person to another; it will be then important to study the relationship between individual specificities and effectiveness of regulation strategies of regret. It is also recommended for future research to explore the circumstances under which these strategies of regulation will have a reducing effect on the intensity of postpurchase regret.

\section{References}

Aron, D. (2000). 'Coping With the Purchase Outcome: Distinguishing Consumer Dissatisfaction from Consumer Regret,' Amarican Marketing Association, Conference Proceedings, Vol. 11, pp. 84-85.

Baggozzi, R. P., Baumgartner, H., Pieters, R. \& Zeelenberg, M. (2000). "The Role of Emotions in Goal-Directed Behavior," In S. Ratneshwar, D. G. M., C., Huffman, (Eds.), "The Why of Consumption", pp. 36-58, London: Routledge.

Carver, C. S. \& Scheier, M. F. (1998). On the Self-Regulation of Behavior, Cambridge, UK: Cambridge.

Gilovich, T. \& Medevec, V. (1995). "The Experience of Regret: What, When an Why," Psychological Review, Vol.112, pp.379-395. 
Shepperd, J. A., Findley-Klein, C., Kwavanick, K. D., Walker, D. \& Perez, S. (2000). "Bracing for Loss," Journal of Personality and Social Psychology, Vol. 78, pp. 620-634.

Van Dijk, W. W., Zeelenberg, M. \& Van der Pligt, J. (2003). "Blessed Are They Who Expect Nothing: Lowering Expectations as a Way of Avoiding Disappointment," Journal of Economic Psychology, Vol. 24, pp. 505-516.

Zeelenberg, M. \& Pieters, R. (1999). "Comparing Service Delivery to What Might Have Been Behavioral Responses to Regret and Disappointment," Journal of Service Research, Vol. 2, N․ 1, pp. 86-97.

Zeelenberg, M. \& Pieters, R. (2004). "Consequences of Regret Aversion in Real Life: the Case of Dutch Postcode Lottery," Organizational Behavior and Human Decision Processes, Vol. 93, pp. 155-168.

Zeelenberg, M. \& Pieters, R. (2007). "A Theory of Regret Regulation 1.0," Journal of Consumer Psychology, Vol.17, pp.3-18.

Zeelenberg, M., Van Dijk, W. W.,Vvan der Pligt, J., Manstead, A. S. R., Van Empelen, P. \& Reinderman, D. (1998). "Emotional Reactions to the Cutcomes of Decisions: the Role of Counterfactual Thought in the Experience of Regret and Disappointment," Organizational Behavior and Human Decision Processes, Vol.75, pp. 117-141. 